Euskal ikerketen aldizkaria | Revue d'études basques |

Revista de estudios vascos | Basque studies review

19 | 2016

Numéro XIX

\title{
Pèir de Garros et la Rhétorique
}

\section{Christian Bonnet}

\section{OpenEdition}

Journals

Édition électronique

URL : https://journals.openedition.org/lapurdum/3214

DOI : $10.4000 /$ lapurdum.3214

ISSN : 1965-0655

Éditeur

IKER

Édition imprimée

Date de publication : 1 janvier 2016

Pagination : 121-131

ISBN : 978-2-95534-134-6

ISSN : $1273-3830$

\section{Référence électronique}

Christian Bonnet, « Pèir de Garros et la Rhétorique », Lapurdum [En ligne], 19 | 2016, mis en ligne le 01 janvier 2021, consulté le 03 septembre 2021. URL : http://journals.openedition.org/lapurdum/3214 ; DOI : https://doi.org/10.4000/lapurdum.3214

Creative Commons - Attribution - Pas d'Utilisation Commerciale - Pas de Modification 4.0 International - CC BY-NC-ND 4.0 


\section{Pèir de Garros et la Rhétorique}

Christian BONNET

Université Blaise Pascal (Clermont II), CERHAC

Le regain d'intérêt public pour Pèir de Garros, dont témoignent les récentes éditions de P. Guilhemjoan et C. Labandés ou de Jean Penent, succédant aux progrès de quelques décennies d'études seiziémistes occitanes depuis les pages initiatrices de Robert Lafont, jalonnées notamment par les travaux de Ph. Gardy, R. Darrigrand ou J.-F. Courouau, invitent à une étude à frais nouveau du grand poète gascon, dont la thèse de G. Couffignal vient de rappeler l'importance et l'actualité.

L'étude d'un auteur si peu renseigné et encore mal connu, oblige nécessairement à en privilégier le texte et, restant tributaire de l'évolution générale de l'herméneutique et l'historiographie littéraires, ne peut avancer qu'avec prudence. Ayant eu l'occasion de porter un regard sur la paraphrase des Psaumes de David par le Lectourois, je voudrais proposer aujourd'hui, très modestement, quelques observations sur son rapport à la rhétorique, à partir du célèbre plaidoyer de son Epistola III en faveur de la langue natale. Je suis particulièrement heureux de les adresser, avec un très amical respect, à un humaniste et protecteur des langues tel que Xarles Videgain.

Quiconque parcourt les lignes vibrantes de la célèbre lettre est sensible à son effet oratoire, et nul ne réduirait à la seule dimension poétique ce texte en octosyllabes à rimes plates, cependant consacré à l'écriture de la fiction littéraire en la forme jugée plénière de son temps, à savoir versifiée. Publiée dans le recueil des Poesias gasconas, 1567, second œuvre poétique du Réformé Garros, cette épître fait partie d'un groupe de quatre, parmi les pièces finales. Elle est adressée «Au medish », soit 'au même' interlocuteur que les deux premières, un certain H. B. en qui l'on a jadis proposé de voir le poète, un temps organiste de la cathédrale de Lectoure, Hector de Beaulieu, en dépit de son origine limousine. La présumée réponse de Garros est en effet contemporaine de l'acmé du processus d'expansion écrite des langues dites vulgaires, mais non toutes nationales, à la modernité duquel il souhaite associer son occitan gascon. Le caractère argumentatif et la cohérence de ce texte sémantiquement fort, engagé dirions-nous, font communément consensus. Il parait donc intéressant d'examiner les 
éléments qui lui confèrent cette force, d'intention toute rationnelle, on ne peut plus étrangers aux conceptions poétiques qu'introduira le Romantisme.

Sans vouloir entreprendre une étude stylistique, nous relèverons d'abord quelques emplois de procédés rhétoriques. On se souvient que la rhétorique appartient au corps d'éducation classique du trivium, où elle prend place entre la grammaire et la logique, maîtrise du discours joignant celle de la langue à celle de l'intellect. Ce trio constitue la base de toute formation postérieure à l'alphabétisation et va de pair avec l'étude du latin, langue d'enseignement et seule enseignée, dans laquelle sont rédigés les traités. Les études juridiques de Garros à l'Université de Toulouse, ainsi que ses débuts poétiques au 'Collège de rhétorique' de cette ville, héritier des Jeux Floraux troubadouresques, disent déjà que le jeune humaniste ne dut rien ignorer des arts de bien dire, soit par parole ou par écrit, au moment où renaissaient les études latines. La première considération de qui veut par le verbe susciter persuasion, réflexion ou plaisir chez autrui est le choix du sujet et de son approche, ou pour employer le terme rhétorique, l'invention de son propos. Et c'est le premier point auquel l'auditeur / lecteur de notre épître est sensible, pour son originalité. La valeur de la langue vulgaire, défendue depuis longtemps par les Italiens, n'est plus un thème nouveau chez les Français mais a surtout donné lieu à des développements dissertés, rédigés en prose. Ainsi fait Du Bellay dans la première partie de sa Deffence et illustration, 1549, paraphrasant servilement l'Italien Speroni, qui pourtant avait composé sous la forme plus animée de dialogues son bilan pro et contra. En cent octosyllabes, Garros va transformer la confrontation, héritée du vieux débat médiéval, en un dialogisme concertant.

Le texte met en scène deux actants, confrères en poésie, dans une connivence à caractère illocutoire : "Puish doncas que plasut vos a (...) de mi sollicitat», vers 1-3: de vous à moi donc, et de la sollicitation à l'action : un poète répond en vers aux vers adressés par un confrère. Structure doublement ingénieuse qui d'une part place en abyme l'acte versificatoire, de l'autre renvoie spéculairement l'écrivant à un alter ego, auquel ne manque qu'une parole propre pour constituer une prosopopée, figure qui cependant parcourt le texte de bout en bout. A travers la langue en partage est ainsi promptement franchi le pas conduisant de la connivence à une complicité générale : "Tots acordats e d’ua conspiracion bandats », vers 27-28. Et voici l'auditeur, indifféremment lecteur en l'absence de l'un comme l'autre interlocuteur (la seule réalité concrète étant le texte), passé à son insu du statut de témoin à celui de coopérant, sinon de coauteur entraîné dans un élan verbal performatif! Mais la force de conviction de cette pièce fameuse s'autorise d'emblée d'une habile mise en œuvre des mécanismes littéraires de la représentation, dont les ressources sont manifestement familières à l'auteur.

La seule assignation de plaidoyer est-elle dès lors suffisante? Le texte tresse en fait deux thèses successivement dominantes. D'abord est présentée (1-42) la situation de déréliction de la langue damnada, maudite semble-t-il de ses défenseurs naturels. La veëmencia explicite (v. 25) du ton porte condamnation des fautifs: la communauté toute entière « Nosauts medish nos trufam d'èra », 'nous-mêmes (qui) nous gaussons d'elle'. C'est la dérobade malhonnête d'une famille dénaturée, désormais indigne du paradis langagier et méritant l'expulsion : « liatge abusat / digne d'este despaïsat », v. 11-14 où le mot 'lignée' (bientôt suivi d' éducation', 16) parait viser particulièrement la jeune génération. Malgré un présent de narration, la prédominance temporelle est au passé : la faute est consommée, qui appelle d'ailleurs réparation, avec basculement final au futur : "Armèm nos / per qu'om presique d'atge en atge » : 'armons nous (de plumes) pour qu'on prône d'âge en age (la victoire de la parole)'. Le ton, non exempt de 
connotations religieuses, est, on le comprend, celui du genre rhétorique qualifié de judiciaire.

La seconde partie (vers 43-90, avant une courte péroraison) offre un contraste édifiant, d'une tonalité autrement plus paisible. Elle se partage entre la félicité (« lo cor gadau », v. 49 : le cour réjoui) du redressement promis par l'entrée en lice d'un second champion (futur proche: «Aras cau díser / la sason èster » 55-56: 'on peut maintenant dire la saison venue', c'est le premier pas qui coûte), et la peinture circonstanciée d'une longue ère de félicité gasconne originelle, située dans un passé très lointain (en fait mythique, donc intemporel). On reconnaît ici les caractères temporels des genres délibératif et surtout épidictique de la rhétorique, dans lequel le thème de l'âge d'or rejoint l'éloge des valeurs sociales (la mémoire historique, les sciences et les lettres). Garros fonde la cohérence et par là le sérieux de son propos sur la mise en œuvre du puissant moteur discursif du 'lieu du possible', point de transit argumentaire commun aux trois genres, selon lequel ce qui a été fait peut être défait et le perdu retrouvé. Linversion de la déchéance en redressement, du déclin en renouveau, ne pouvait manquer de rappeler au juriste le concept de parallélisme des formes, ni au Réformé le contraste du mal et du remède, sinon de la chute et de la rédemption : "Los libes doncas son perits/ Mes non pas los bons esperits / Qui las pècas corregiràn / .../ Si vos avètz ja començat. » ('les œuvres ont péri mais non les hommes pour y remédier, comme vous commencez', v. 91-95). Ce procédé permet aussi d'instruire contradictoirement le lecteur / auditeur des éléments du débat, fonction nécessaire du docere rhétorique.

La seconde tâche que s'assigne l'orateur (fut-ce in absentia, pour l'épistolier) est d'ordonner son propos. La tradition rhétorique emploie ici le terme de dispositio, qui ne se limite point à l'ordre de succession, mais englobe la sélection et le traitement des arguments. L' introduction d'un interlocuteur de même opinion permet à Garros d'abréger l'exorde, moment initial de conciliation avec les auditeurs, indispensable au prétoire. Il n'en néglige pour autant aucune des suavités, de la laudatio du correspondant alludant à celle du lecteur (« plasut vos a compausar»= 'qu'il vous plaise entendre', v. 1-6), à l'excusatio propter infirmitatem, excuse de maladresse de l'orateur plaidant la suppléance de ses défauts par la bienveillance de l'auditeur, ou de l'avocat invitant le juge à recevoir une action mal présentée en reformulant à son aune le moyen soulevé. On est alors proprement, vers 51-54, dans la figure du chleuasme, mécanisme d'auto-dénigrement rendu ostensible, visant à de subtils effets psychologiques sur les témoins. Ici, le récipiendaire remercie son correspondant moins pour les compliments glissés dans sa réponse, Garros protestant ne pas mériter l'attribut de bon poète, que pour la qualité supérieure des vers qui la formulent : "Non pas per autant qu'era'm lausa, / Perque jo sabi que sens causa / M'avetz bon poèta estimat, / Mès (per que) vos avetz tan plan rimat. ». Sous cette posture de modestie, notre adroit auteur introduit la preuve interne d'une aptitude poétique positive, assurance éthique d'une autorité probable de son point de vue.

Cet avocat ou poète, s'il minore sa réputation, en pose donc l'existence, qui renvoie autant à la figure présumable de Garros qu'à l'assentiment supposé du lecteur, placé en témoin indiscret de l'échange. Ce jeu de dépréciation valorisante constitue donc un autre lieu (de l'existence ou l'inexistence) par lequel s'administre une preuve logique désormais certaine : en poésie la langue gasconne n'est pas dépourvue de man mestressa ('main de maître' est celle du correspondant, v. 47), sinon implicitement de deux. On mesure avec quelle habileté l'image de la lettre reçue, que pourtant on ne voit point, se trouve ainsi assimilée à une poésie dûment existante. Par ce stratagème, la présumée réponse (que Garros prend soin de faire suivre de l'épître d'un tiers, donc reçue et a priori authentique, seule pièce exogène du volume) peut 
glisser du statut de preuve interne au discours (il y a bien poésie puisque faite de rythmes, désignation des vers néanmoins assertive), c'est-à-dire relevant de la technique discursive (et de la bonne foi de l'orateur), à la fonction de preuve matérielle externe (dite extra-technique par le rhétoricien), relevant de l'ordre des réalités physiques et dès lors irréfutable (hors de débat). Oratoire ou littéraire, Garros a de son art une maîtrise consommée.

Nous pourrions observer encore diverses figures de construction, comme la prompte succession narratio - confirmatio - refutatio de la première partie, faisant ressortir les périodes oratoires, ponctuées de clausules restées fameuses : «Aquo b'es ... Son pais mau recompensar »v. 21-22, «Per l'onor deu país ... E sa dignitat manténguer » v. 29-30, « La gent tan bèra parladora / Coma ... vencedora ». De même, la longue énallage de la deuxième partie, déjà évoquée, alterne des verbes fléchis aux trois temps indicatifs, présent, passé et futur, ainsi qu'au subjonctif, pour référer à des périodes alternativement proches ou lointaines de passés, tantôt historiques ou légendaires, qu'un futur hyperbolique promet finalement à l'apothéose. C'est qu'il ne s'agit de rien moins que d'asseoir l'audacieuse assertion d'une antériorité de la culture gasconne sur celle de l'Antiquité gréco-latine.

Le déploiement de moyens rhétoriques opéré par Garros, qu’amplifie lexicalement la séduction d'images d'une prospérité idéalisée, en dit long sur le prix qu'il attachait à cette belle ambition, que les Français avaient déjà défendue à qui mieux mieux en faveur d'une précellence gauloise. Sans alluder à celle-ci, le Lectourois pose le fondement d'une alternative intinsèquement gasconne à la délivrance des Modernes de la dépendance classique, faisant d'une pierre deux coups : dotés d'un patrimoine propre aussi prestigieux, les Gascons n'ont plus que faire d'une langue vulgaire étrangère pour entrer dans la modernité. Le recours au français est donc une parodie (" un lengatge hardat », v. 18) au prétexte fallacieux. Nous sommes là au moment du placere, où l'orateur tente de rallier à sa thèse l'auditeur mis en mesure d'en reconnaître le bien-fondé. Il est significatif que le critère esthétique y contamine l'exigence d'efficacité discursive, dans une parfaite appropriation au sujet (aptum).

Nous pouvons encore suivre l'empreinte de la rhétorique dans le détail de la formulation, domaine du troisième volet de l'art rhétorique, l'elocutio. Sa marque s'observe dans de nombreuses figures de construction, de la tmèse (rupture solennisante de l'ordre syntagmatique : 'puis, donques, qu'il vous a plu...'v. 1), aux répétitions en écho ('armons nous... pour orner le gascon ... pour qu'on vante...' v. 38-40) ou enchâssées ('...pour qu'on vante d'âge en âge' v. 40, allusion à la théorie cyclique des âges historiques) voire embrassées ('vous avez commencé' 95 / 'bon commencement' 97 'bon achèvement' 98 / 'aucun achèvement' 100, avec anadiplose). Le lexique fournit évidemment une contribution capitale à ce stade, soit que se redoublent des sémantismes forts (honneur / dignité, 29-30) soit que des antagonismes se répondent à distance (véhémence / nonchalance, 25-89 ; soutenir / déchéance, 29-90).

On relève sans surprise un nombre conséquent de termes à résonance morale (lignée, aïeux, éducation «noiritud », soutien, protection «(des)-empara », abus, ingratitude, récompenser, digne /-ité, religieusement), militaire (dont une belle métaphore filée assimilant 'la lance et la plume', guerre et écriture, comme inscriptions parallèles de l'histoire, vers 3142), juridique (prendre cause, défendre, soutenir, maintenir, jurer, user d'échappatoire, tenir compte, mettre d'accord) ou politique (exilé, conspiration, liguer, triompher, conquérir) et notamment la notion de droit naturel ("l'ajuda au país naturau deguda » 'laide due au pays naturel', v. 19-20). Le sémantisme très appuyé de ces séries souligne l'ultime apport du movere 
à la visée morale de cette tentative de persuasion. Qui nous émeut ne peut avoir tort : le rhétoricien parle de preuve pathétique.

Garros est donc amplement pourvu des moyens oratoires et, passant à l'écrit, stylistiques de son entreprise. Le reste du volume atteste d'ailleurs d'une culture littéraire étendue. Observons cependant le lexique correspondant à ce domaine. C'est une série plus discrète parce qu'assimilée, dans la première partie, aux termes mêmes de la revendication linguistique, ou associée dans la seconde aux éléments du pittoresque historisant. Les rythmes en gascon composés désignant la réponse emploient les termes mêmes caractérisant l'expression poétique : mise en forme versifiée, langue propre, formulation travaillée. A barbare ('sauvage', 10) s'oppose ensuite la noiritud, v. 16, sens fort de 'nourriture' qui réunit la double acception d'aliment (nutritionnel et intellectuel, cf. 'nourri de latin') au sens figuré de 'éducation morale et culturelle' (cf. 'nourrisson des muses', 'nouyrigat de Toulouso' chez Goudouli). Ce terme, précédant celui de lengatge hardat, v. 18, attire l'attention sur la différence établie par Garros entre 'langue naturelle' (nosta lenga mespresada, méprisée, 6) et 'langage' entendu comme artefact, 'outil ou façon de communiquer' : le langage 'fardé' se voyant dès lors soupçonné non seulement de fallace, mais de dévoiement, comme 'nourriture frelatée', impropre à élever la jeune lignée. Or lengatge se retrouve (relayé, v. 25, par un performatif jo scriurè : mais moi 'j'écrirai') en conclusion de la première partie, v. 39, plaçant cette fois le sémantisme d'élaboration délibérée' au service d'une écriture culte du gascon : "Armèm nos de plumas agudas / Per ornar lo gascon lengatge », qui rendra ce 'peuple aussi éloquent qu'il est en armes victorieux" "gent tan bèra parladora coma en armas vencedora », 41-42.

La seconde partie de la pièce emploie d'abord le mot d'incitament 'incitation', v. 45, qui prolonge la référence à l'émulation littéraire en rappelant pour mieux s'en féliciter la réponse reçue. Profit non négligeable, non pas petit, assurément, puisque 'une main maîtresse une lettre rimée m'adresse', v. 48. C'est donc un texte en vers, forme supérieure de l'écriture littéraire (pour lors, la prose n'est encore consacrée que dans la nouvelle), composé (discursivement) de main de maître, où non seulement le narrateur est complimenté (me lausa, 51) pour sa propre production (M'avètz bon poëta estimat, 54), mais si excellemment versifié « tan plan rhytmat en gascon » qu'il couronne véritablement son effort prosélyte " (coma) si hos ua corona d'aur». 'Voici la saison venue' (la preuve en est faite " eth cau díser », 'il faut le proclamer') pour la 'langue' gasconne (58, supérant ici le langage) de retrouver le statut (en tau punt tornada) où ses Anciens l'avaient haussée. On note la valorisation croissante qui accompagne l'entrée en scène de ces derniers: dans cette époque idyllique le gascon avait été langue de l'astronomie, de la géographie, de l'histoire et de l'économie, joliment évoquées (l'ornamentum d'un style médian ayant succédé à la vivacité attique). Aussi bien 'régnait-elle et sur toutes autres triomphait', v. 78: Columelle et les Latins n'ont eu que la peine de traduire! On a situé plus haut l'enjeu d'un tel développement, qui n'est pas de pure gloire.

Arrivés à la péroraison, une dernière série fait encore explicitement référence à l'activité littéraire. Les 'lettres amoureuses' des aïeux, v. 80-81, ne visent aucunement leur correspondance mais clairement cette production lettrée, dont il convient de décrypter la qualification d'amoureuse, qui n'est évidemment pas d'ordre sentimental. Les 'anciens livres' (v. 88, mot répété v. 91 pour les constater perits 'perdus par destruction'), ceux des bons Anciens donc, confiés à la posteritat 79, à charge de les continuer et compléter, ont été fautivement délaissés au profit d'activités guerrières (allusion à la réputation gasconne illustrée dans les Eglogas). Nous tenterons de revenir ailleurs sur la question de ces mystérieux 
ouvrages. Mais si ces générations avaient délaissé l'écriture, du moins eussent-elles du en assurer la garde, v. 87-88 ! Cette évocation inversée du gradus ad Parnassum annonce le verdict final de descasença, 'déchéance / décadence', v. 90, ultime rappel du péril conjuré in extremis et du 'recommencement' promis par l'effort. Car si les livres sont perdus, restent « los bons esprits qui las pècas corregiràn quan voleràn », de 'bons esprits propres à combler les manques quant il leur plaira', 91-94. Eux sont présents, et l'ami ouvre bénévolement le chantier. Une 'gloire éternelle' (avec quelque emphase du style élevé) restera ainsi acquise à ce correspondant secourable, dont il est néanmoins brusquement pris congé. Pourtant, ni le caractère ostensible de 'morceau d'éloquence', ni ce finale proche du sublime ne peuvent exclure la réalité d'un véritable échange épistolaire avec $\mathrm{H}$. B.

Mais quelques mots nous retiennent encore. On remarque dans cette Epistòla le statut privilégié, tout d'héritage classique, fait à la poésie versifiée, sommet du Parnasse. Il permet une banalisation du terme de rythme, employé à trois reprises (vers 2, 48, 54) au détriment de 'vers'. Utilisé par les humanistes pour désigner l'écriture versifiée, il parait aujourd'hui pédant, mais revêtait bien à l'époque une acception poétique spécialisée. Son emploi dénote une attention portée par Garros à la théorie poétique. Vient alors l'évocation, v. 61-63, d'une créance circulant en la matière, érudite donc : "Òm ditz que nòstes bons Antics / Nos avèn, en nombres poetics / Descriut lo Cèu empireau » 'On rapporte que nos bons Anciens, avaient décrit le Ciel empyrée en nombres poétiques'. Ladjectif, en excluant toute numération, rattache la locution au même domaine intellectuel. Sans doute est-ce encore celui des 'bons esprits' qui travaillent aujourd'hui à combler les lacunes, corriger les manques, composer des épîtres. Il y a enfin le nom propre de 'Columelle' : Lucius Junius Columella auteur latin d'un important traité d'agronomie De re rustica. C'est le coupable désigné par Garros pour avoir traslatada, 'transposée' en latin la maîtrise des Gascons « en la causa rustica», vers 73-76. La reprise exacte du titre rejoint la précision du nom : mais qu'avait affaire le rhéteur et juriste gascon de douze livres latins sur l'agriculture?

On sait qu'à l'instar de son illustre prédécesseur à Toulouse, le jurisconsulte et poète Boyssoné, Garros avait étroitement associé l'étude du droit et celle des lettres. Il serait évidemment précieux d'être davantage renseignés sur ces études. Les décennies précédant immédiatement la parution des Poesias, en 1567 sont en effet celles de la célèbre querelle du cicéronianisme. Le fond n'en est ici aucunement indifférent puisqu'il concerne, avec l'orateur romain, le maître et théoricien majeur de l'art rhétorique en même temps qu'un lointain précurseur du processus d'émancipation qui anime les langues romanes depuis les troubadours. En effet, Cicéron n'avait pas seulement porté au sommet l'éloquence latine, il avait, ce faisant, entendu montrer que l'expressivité de la langue latine ne le cédait en rien au modèle grec et devait désormais avoir préférence sur lui. Aussi les humanistes italiens, remettant précocement en honneur l'héritage classique s'étaient-ils interrogés, de Dante à Bembo, sur la façon de suivre leur modèle (ils emploient le mot d'imitatio), soit dans un culte exclusif de la pureté latine, soit dans la recherche d'une éloquence vulgaire de même exigence. Comment être fidèle ('à la hauteur du modèle') en imitant Cicéron ?

Le jeune humaniste français, Etienne Dolet se saisit de la question en 1535, dans un dialogue De Imitatione Ciceroniana, suffisamment engagé dans la voie puriste pour oser 
reprendre le Ciceronianus (1528) du vénérable Erasme. Lui reprochant insolemment sa tiédeur cicéronienne, Dolet fit justement du nombre le critère de perfection décisif qui élève Cicéron au-dessus de tous les Anciens. Or le nombre (numerus) était pour les rhétoriciens latins l'équivalent du grec rythmos, points concernant la scansion oratoire et rejoignant la notion de 'période' (développement phrastique complexe, mais présentant une unité rythmique). Le détail qui nous intéresse maintenant est biographique : l'orléanais Dolet venait précisément d'achever sa formation à Toulouse, fort brusquement. Etudiant, élu syndic de la 'nation française' de l'université languedocienne, il avait d'abord engagé une violente querelle avec le syndic de la 'nation gasconne', suscitant des troubles entre les deux corporations (à recrutement géographique, mais par là à résonance linguistique). Il eut ensuite des différents tels avec le milieu littéraire local, encore fidèle à la 'seconde rhétorique' (désignation ancienne de l'écriture versifiée, la 'première' étant la rhétorique oratoire), qu'il dut s'enfuir à Lyon poursuivre la carrière prestigieuse et funeste que l'on sait.

Or un passage de son ouvrage attire ainsi l'attention : «Latinè loquatur Livius, Plinius uterque, Quintilianus, Gellius, Columella, Vegetius, Vitruvius, Seneca : non aliis tamen verbis, atque Cicero, utuntur, sed aliter compositis, hiulcè saepe $\&$ vastè contextis, nec ad numeros latinos, \& germanam linguae latinae phrasim cadentibus » (De Imitatione Ciceroniana adversus D. Erasmum, Lyon, S. Gryphe, 1535, p. 59 ; cité par Meerhoff 1986 : 28). De la générosité du verbe, la richesse de l'inspiration ou la maîtrise de la composition, à laquelle le nombre confère une distinction supérieure, la composition est la plus importante, en quoi Cicéron l'emporte sur les autres modèles. Naturellement on s'interroge sur la présence du 'rustique' Columelle dans cet aréopage. Lagronome du premier siècle, auteur de la somme encyclopédique déjà citée, ne se consolait pas du mutisme observé par le grand Virgile, au sujet des jardins, dans ses Géorgiques. Pour réparer ce malheur, il composa en vers son dixième livre, consacré à l'art des jardins, prenant talentueusement le Mantouan pour modèle. Lecteur obligé de Virgile, au cœur d'une région agricole prospère, Garros aura connu le fait pour son compte. Certainement informé du passé latin de sa région, il lui fut aisé de penser que la mise en valeur de celle-ci sur ce plan n'avait pas attendu l'arrivée des légions. Et de franchir le pas de l'antériorité gasconne.

Le différend entre les cicéroniens 'purs' (au rang desquels il faut ajouter le voisin agenais Jules-César Scaliger) et les autres auteurs néo-latins à la suite d'Erasme, est que ce dernier, observant les profondes différences de réalisation orale du latin selon les pays, avait bien reconnu qu'il n'était plus prononcé comme au temps de Cicéron et que le sentiment de la quantité vocalique ('longues' et 'brèves', essentielles à la métrique latine, les pieds) s'était perdu. Cela rendait inopérant dans les langues vulgaires le savant système de décompte qui en était découlé (numerus copiosus). Autant, pour Erasme, employer son talent oratoire en vulgaire roman (dans lequel le phonologisme latin s'est transformé en rythme accentuel tonique). Dolet, notre paradoxal toulousain et puriste, dut en convenir par une volte-face : La manière de bien traduire d'une langue en aultre, Lyon, 1540, significativement rédigée en français. Le pédagogue et penseur humaniste dominant après Erasme, Jean Ramus, confortera cette conséquence logique à partir de 1556 ; évasif sur le nombre dans son traité de rhétorique Ciceronianus, 1557, il bascule ensuite vers l'emploi du français.

La théorie de l'imitation qu'avait actualisée la querelle du cicéronianisme, se vit reconvertie en concept d’innutrition, emprunté à Quintilien, par les défenseurs des langues vulgaires. Ainsi Du Bellay employant le verbe 'dévorer' pour décrire l'absorption par les Romains de la culture des Grecs, « et après les avoir bien digérez, les convertissant en sang et nourriture », Deffence 
et ill., I, 7. On reconnaît l'image biologique aperçue chez Garros, qui la reliait peut-être à la textologie chrétienne. Le Rémois Strébée (Strebaeus, en fait Jacques Estrebay), cicéronien réservé, avait par exemple opté à la suite d'Erasme, dans un traité rhétorique de 1538 De electione, contre la rétro-conversion des néologismes de source chrétienne en termes païens (tels que 'Dieu le Père' en 'Jupiter'). Lui aussi étudie la question du nombre, qu'il tente de démêler, faisant cas des effets sonores, concevant la présence d'une métrique dans la prose, en particulier en fin de clausule, mais reprochant à la poésie vulgaire de réduire la rime à une figure de diction, trop monotone. De telles préoccupations ne devaient pas être étrangères à Garros si l'on compare la sobriété de l'Epistòla à la versification travaillée de son Cant noviau. Mais à la différence des Cicéroniens, il privilégie la poésie comme achèvement esthétique de l'écriture littéraire, propre à mieux consacrer l'émancipation de l'écriture gasconne.

Pour les nouvelles motivations de Dolet à Lyon, leur caractère nationalitaire s'inscrit pourtant dans le droit fil de ses démêlés toulousains : " Mon affection est telle envers l'honneur de mon païs que je veulx trouver tout moyen de l'illustrer, \& ne puis myeulx faire que de célébrer sa langue comme ont faict Grecs et Rommains la leur» (dédicace de la Manière de traduire, l'ouvrage étant placé sous le patronage de l'influente famille Du Bellay en la personne de Guillaume). On est sensible à la proximité des termes qu'emploiera Garros: outre le soutien envers l'honneur du pays, Dolet veut œuvrer «à telle fin que les estrangers ne nous appelleront plus barbares », formulation dans laquelle le pluriel du sujet confronte sa langue à la diversité babélienne. Alors que J. Du Bellay recourt au singulier pour isoler le seul français « Que la langue françoyse ne doit estre nommée barbare » (Deffence, titre du ch. II), une même confrontation à la multitude est présente chez Garros : "Cad'un la leisha, tot lo mond l'apera barbara », v. 9-10, 'chacun / tout le monde', malgré le glissement opéré dans les sujets, de chaque, visant les membres de la communauté natale (le pays), au monde, dans les révolutions duquel elle s'engloutit.

Si les règles techniques dégagées dans la Manière restent tirées de Cicéron, la cinquième s'en détache pour traiter de l'harmonie du style en français, que Dolet attribue à l'observation des nombres en prose, c'est-à-dire à l'emploi stylé des périodes. Pour être élégante, toute composition doit selon lui observer «les nombres oratoires, c'est à sçavoir une liaison et assemblement des dictions avec telle doulceur [harmonie] que non seulement l'âme mais les oreilles en sont toutes ravies », faute de quoi « les sentences ne peuvent être graves et avoir leur poix [avoir la majesté ni l'effet] requis et légitime. Car (...) c'est peu de la splendeur des mots si l'ordre et collocation d'yceulx n'est telle qu'il appartient ». En un mot : "Sans grande observation des nombres, un autheur n'est rien »! Quelle autre donc pourrait être la préoccupation du 'presque bon poète Garros', que la décence empêche de s'autoproclamer 'maître auteur', au profit de l'ami ? En bon cicéronien, Garros est ingénieux : si le Gascon est soldat de race, le Poète est virtuose de 'nature' : les nombres lui sont héritage de famille ! Et d'imiter au profit du pays le dépassement des Grecs par le modèle Latin.

Pour préciser son idée force, Dolet ajoute un appendice sur la ponctuation dans lequel il définit la période comme "un terme grec que les Latins appellent clausule ou groupe de paroles, segmenté et subdivisé par le point et les deux points [équivalent à notre ; ] et qui « communément ne doibt avoir que deux ou trois membres, car si par la longueur, il excède l'halène de l'homme, il est vicieux». Malgré cette inclusion de la performance oratoire (souffle, harmonie), cette vue est surtout adossée à la syntaxe et aux figures de mots : antithèses, anaphores, intonations (interrogation, exclamation), métrique accentuelle (pour le cadencement tonique). C'était, sans véritablement définir le nombre oratoire français, et pour cause, translater au 
mieux la concinnitas latine, l'agencement le plus ingénieux de la phrase tendant à l'harmonie et à la séduction. Tels sont aussi, de toute évidence, les moyens déployés par Garros dans son épître, dialogue inavoué qui place le lecteur au cour d'une conversation élevée, dans la vive succession des idées, la franchise sans détour des opinions, la générosité poétique du développement final, cette « vis numerosa quibus versus clauduntur » prônée par Jules-C. Scaliger.

La revendication française de Dolet, favorisée par la politique du pouvoir royal Valois, rencontra l'approbation des poéticiens et l'ouvrage ne connut pas moins de cinq rééditions de 1540 à 1548. Est-il concevable de penser que Garros, toulousain d'éducation et Gascon par inclination, avocat et poète, voisin lectourois de Scaliger, ait eu dans les deux décennies suivantes quelque écho du personnage ou connaissance de ses ouvrages ? Observons que Toulouse, entre influences française et italienne, est une pépinière de rhétoriciens militants. Dès 1523, Guillaume Lelièvre y publie son Ars memorativa, ouvrage didactique spécialement dévolu à la quatrième technique rhétorique, celle de la memoria. Il y présente, à l'aide de savantes figures typographiques, toutes les combinaisons mnémotechniques permettant à l'orateur de se remémorer en détail et sans note toute la teneur de son discours, mimiques incluses. En 1526, Jean Maur, qui avait auparavant dirigé les écoles de Lectoure, publie son In 'Chiliades adagiarum' D. Erasmi compendiosa espositio, qui étudie les figures rhétoriques dans le recueil de proverbes latins publié par Erasme. Vers 1534, la correspondance de Boyssoné bruit de questions de rhétorique poétique. En 1536 Robert Breton, qui avait d'abord professé à Bordeaux au Collège de Guyenne, épigone parisien, publie des Orationes quatuor, contenant à la suite trois livres d'épîtres. Il s'y interroge sur la primauté à donner au latin ou aux langues vernaculaires.

Persécuteur de Dolet, Du Pont de Drusac qui suit encore Fabri, franchit le pas du français en 1539 avec son Art et science de rhétorique métriffiée, par ailleurs plein de menaces envers les évangéliques. A partir de 1538, l'humaniste toulousain Pierre Bunel, proche des Du Faur, passe pour un modèle d'épistolier latin, cicéronien naturellement, que les Estienne éditent à Paris après sa mort : P. B. familiares aliquot epistolae in adolescentorum Ciceronis studiorum gratiem, 1551, puis P. B. praeceptoris... epistolae, ciceronianae stylo scriptae, 1581. En 1541, Pierre Lagnier, autre ancien du Collège de Guyenne, publie son manuel M.T. Ciceronis elegans ac perutile compendium. Un plus modeste ouvrage retient ici notre attention, celui du juriste Jacques Nariguan, paru chez Guyon Boudeville à Toulouse, en 1554. Intitulé De Oratore civiliter instituendo, liber singularis methodicem et compendiosem descriptus, c'est un court guide méthodique de l'avocat (92 pages de la taille d'un in 12), dont la rhétorique constitue le fond.

Cet opuscule dédié à l'évêque de Lectoure nous renseigne sur la famille de Garros pour contenir à la fin une épître latine signée de Pèir que la suscription adresse à son oncle maternel en la personne du même Nariguan. Le poète s'y déclare redevable de l'enseignement rhétorique de son parent, homme de culture qui cite Vigile et auquel on dédicace une épigramme latine en tête de l'ouvrage. Peut-être cet oncle a-t-il joué quelque rôle dans l'orientation juridique de l'aîné des fils Garros, dont le père était banquier. Ces lignes, qui ne paraissent pas d'un tout jeune homme, sont a priori adressées à une personne de la génération précédente, dont la naissance peut ainsi être placée autour de 1500 et la formation juridique, du moins initiale, à Toulouse. Elles insèrent formellement notre poète dans un réseau de mémoire vivante fort propre à l'avoir renseigné sur les épisodes locaux des années 1535 et suivantes et révèlent par ailleurs une intégration culturelle accomplie au sein de la corporation juridique et du 
milieu humaniste. Attesté comme licencié en 1553, mais à Lectoure et sans référence de date d'obtention, il serait donc possible que sa formation soit sensiblement antérieure. Lattestation comme escolier de Lectoure en 1557, dans l'enceinte toulousaine des Jeux Floraux, ne doit pas induire en erreur : cette formulation vise à marquer la distance des impétrants avec les mainteneurs, aggravée pour lui d'une origine foraine. A la même date, Garros est déjà gratifié ailleurs du titre de docteur.

On est ainsi en droit de penser que Dolet et ses ouvrages aient permis à Garros d'anticiper les réflexions que pouvait susciter une lecture de la Deffence de Du Bellay, à laquelle dut l'inviter plus tard la notoriété de la Pléiade sinon le dédain qu'elle affecta envers Marot. Par ailleurs on peut voir dans son épisode gascon, un écho à la notoriété récemment conférée à l'épisode gaulois par la parution du livre de Ramus, Liber de moribus veterum Gallorum, 1559, aussitôt popularisé par la traduction française de Michel de Castelnau Traicté des façons et coustumes des anciens Galloys. Du reste, Garros récidive dans l'épître l'année suivante avec une nouvelle adresse en prose latine à l'auteur d'un traité sur le droit des servitudes, Jean Superior. Cette contribution confraternelle est révélatrice de l'autorité montante du juriste comme de l'humaniste. On le croira d'autant plus volontiers que Superior dédiait son ouvrage à deux émissaires du duc de Vendôme dont les noms s'inscrivent dans l'histoire de l'humanisme et de l'évangélisme en Gascogne: Mathieu Pac et Claude Regin, à côté de celui de la maison de Navarre en la personne du Vendômois, futur époux de Jeanne d'Albret. Comment Garros aurait-il échappé à son milieu ?

Linsertion d'une section d'Epistòlas dans un recueil poétique n'est sans doute pas anodine après Pétrarque ou Marot et précédant Maynard ou Balzac. Les quelques éléments que nous venons de présenter découvrent une culture littéraire étendue de Garros, dûment informé des apports humanistes contemporains. La mémoire régionale lui était immédiatement accessible par la génération précédente, mais ce praticien et expert confirmé des questions juridiques avait aussi été formé à l'histoire politique et institutionnelle nécessaire aux parlementaires, ainsi qu'à l'étude des documents historiques familière aux feudistes. Il apparaît ainsi le récipiendaire d'une riche tradition humaniste dans laquelle la rhétorique réunit le droit et les lettres dans le culte de la parole et des mours. Suppléant au défaut de renseignements factuels sur ses études et ses débuts de carrière, ces éléments éclairent son ancrage dans ce terreau intellectuel. S'il n'avait pas exhumé le legs troubadouresque conservé au Collège des Jeux Floraux, Garros, en assimilant dans sa poétique l'apport du corpus gréco-latin, épine dorsale de la culture occidentale, réhabilitait dans tous ses droits une littérature gasconne et, par l'inventio d'un passé, dans cette invite superbe, lui permettait d'advenir. 


\section{Réferences}

Bedouret, Sandrine. 2014. Enjeux poétiques du nombre comme élément constitutif du et stylistique: Moyen Age et Renaissance, 47-56. Talence, Presses Universitaires de Bordeaux.

Berry, André. [1948] 1997. L'œuvre de Pey de Garros poète gascon du XVIe siècle : éd. établie par Philippe Gardy et Guy Latry. Talence : Presses Universitaires de Bordeaux.

[Colloque], 1968. Pey de Garros et la situation culturelle de l'Aquitaine méridionale au XVIe siècle. Toulouse, Institut d'Etudes Occitanes.

Garros, Pey de. 1567. Poesias gasconas : dedicadas a Magniphic e poderos Princep, lo Princep de Navarra, son Señor. Tolosa: Iammes Colomes.

Garros, Pey de. 1895. Poésies gasconnes, second volume: traduites du gascon par Alcée Durrieux. Ed. nouvelle. Auch, G. Foix.

Garros, Pèir de. 2001. Epistòla au medish. In Jean-François Courouau, Premiers combats pour la langue occitane: manifestes linguistiques occitans, XVIe-XVIIe siècles, 54-60. Biarritz, Atlantica.

Lafont, Robert. 1970. Renaissance du Sud: essai sur la littérature occitane au temps d'Henri IV. Paris, Gallimard.

Meerhoff, Kees. 1986. Rhétorique et poétique au XVIe siècle en France: Du Bellay, Ramus et les autres. Leiden, Brill.

Penent, Jean. 1980. Pèir de Garros et son temps : la Renaissance littéraire en Occitanie. Toulouse, Menestral.

Penent, Jean (éd.). 1988. Pey de Garros, ca 1525-1583 : actes du colloque de Lectoure, 1981. Béziers, C.I.D.O. 